\title{
NOUVELLE
}

\section{Mécanismes immunologiques impliqués dans la thrombopénie immunologique résistante au rituximab}

Sylvain Audia ${ }^{1}$, Philippe Saas ${ }^{2}$, Bernard Bonnotte ${ }^{1}$
${ }^{1}$ Inserm UMR 1098, équipe immunorégulation et immunopathologie, service de médecine interne et immunologie clinique, CHU le Bocage, boulevard Maréchal de Lattre de Tassigny, 21000 Dijon, France ;

2 Inserm UMR 1098, Établissement français du sang Bourgogne Franche-Comté, LabEX LipSTIC, ANR-11-LABX-0021, 1, boulevard Fleming, BP 1937, 25020 Besançon Cedex, France. sylvain.audia@u-bourgogne.fr

bernard.bonnotte@u-bourgogne.fr philippe.saas@efs.sante.fr
> La thrombopénie immunologique, anciennement nommée purpura thrombopénique idiopathique ou PTI, est une maladie auto-immune rare, spécifique d'organe. La thrombopénie qui résulte de cette réponse immunitaire inadaptée entraîne chez environ 70 \% des malades des manifestations hémorragiques purpura pétéchial ou ecchymotique, ou bulles hémorragiques intrabuccales (Figure 1). Ces dernières constituent un signe de gravité pouvant précéder la survenue d'hémorragies graves, comme les hémorragies intracérébrales. L'incidence du PTI est d'environ $2 / 10^{5}$ individus/an, avec une prévalence de $10 / 10^{5}$. Un traitement est généralement requis lorsque des manifestations hémorragiques sont présentes ou que le nombre de plaquettes est inférieur à 30 giga/l (giga $\left.=10^{9} / \mathrm{I}\right)$. Les traitements de première ligne reposent sur les corticoïdes et les immunoglobulines polyvalentes administrées par voie intraveineuse (IgIV). Utilisés en cures courtes, ils ne permettent qu'une réponse transitoire. Des traitements de seconde ligne sont donc nécessaires, la splénectomie en constituant la pierre angulaire; elle entraîne une guérison prolongée dans $60 \%$ des cas. D'autres thérapeutiques visant à stimuler la production médullaire des plaquettes sont disponibles, mais ne sont réservées en France qu'aux patients réfractaires, c'est-à-dire pour lesquels la splénectomie a échoué, ou lorsque la chirurgie est contre-indiquée. Bien que ne disposant pas d'une autorisation de mise sur le marché dans cette indication, le rituximab, un anti- corps monoclonal chimérique ciblant la molécule CD20 exprimée par les lymphocytes B [1], est largement utilisé et permet d'obtenir une réponse à $l$ an dans $40 \%$ des cas, qui se maintient chez la moitié des patients à 5 ans, ce qui traduit généralement une guérison [2]. Le rituximab est utilisé dans de nombreuses autres maladies auto-immunes ou inflammatoires chroniques [3]. La connaissance de ses mécanismes d'action et la compréhension de ses échecs pourraient par analogie être d'une grande aide dans l'analyse de la physiopathologie d'autres maladies autoimmunes. De plus, le PTI est la seule maladie auto-immune pour laquelle la

splénectomie est indiquée, ce qui permet d'étudier la réponse immunitaire splénique humaine au cours d'une maladie auto-immune chez des patients qui ont reçu ou non du rituximab.

\section{Bases physiopathologiques du PTI}

La baisse du taux de plaquettes résulte de mécanismes intriqués impliquant à la fois leur destruction périphérique et une production médullaire insuffisante [4]. Les lymphocytes B jouent un rôle central en produisant des autoanticorps ciblant différentes glycoprotéines exprimées par les plaquettes (GPIIb/IIla principalement, GPIb/IX et GPIa/IIa). Les plaquettes ainsi opsonisées (c'est-
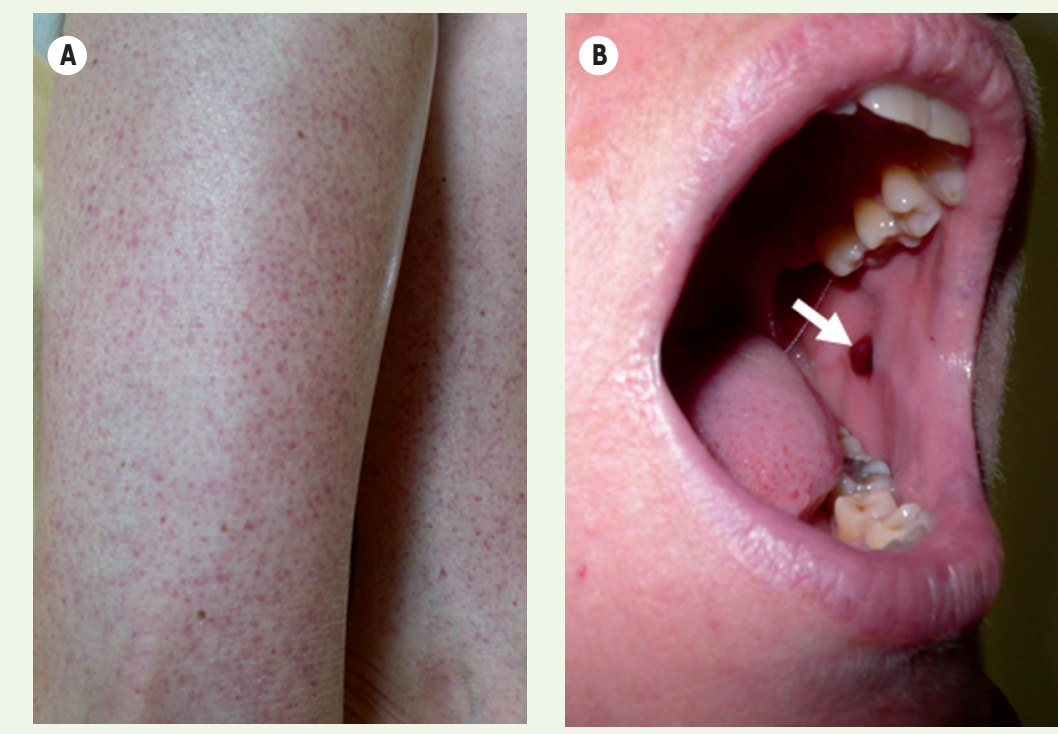

Figure 1. Manifestations cliniques au cours du PTI. A. Purpura pétéchial des membres inférieurs. B. Bulle hémorragique intrabuccale (flèche). 
à-dire recouvertes d'autoanticorps), sont alors phagocytées par les macrophages, principalement au niveau de la rate. Par leur fonction de cellules présentatrices d'antigènes, les macrophages spléniques vont également participer à l'activation des lymphocytes $T$ autoréactifs. Ces lymphocytes T CD4 ${ }^{+}$ participent très probablement à la différenciation des lymphocytes $B$, puisque les anticorps anti-plaquettes sont principalement de type IgG - ce qui traduit une commutation de classe isotypique (IgM/IgG) -, et présentent des mutations somatiques, deux phénomènes dépendant des lymphocytes T CD4. Un déséquilibre de la balance pro-inflammatoire/anti-inflammatoire est également observé au cours du PTI, que reflète une polarisation Thl des lymphocytes $\mathrm{T} \mathrm{CD}^{+}$associée à une diminution de la polarisation Th2 et à un déficit quantitatif et/ou fonctionnel des lymphocytes T régulateurs (Treg). De telles anomalies sont également présentes dans la rate, avec un déficit quantitatif en Treg [5] et une polarisation Th1 [6]. Chez certains patients, les lymphocytes $\mathrm{T} \mathrm{CD}^{+}$participent également à la destruction des plaquettes. Une activité cytotoxique dirigée contre les plaquettes a été observée in vitro, associée à une augmentation de l'expression de perforine et granzyme, deux protéines impliquées dans les mécanismes de cytotoxicité [7].

Associé à la destruction périphérique des plaquettes, un défaut de production médullaire est également impliqué dans le PTI. II résulte en partie de la réponse auto-immune contre les mégacaryocytes (les précurseurs des plaquettes), médiée à la fois par les autoanticorps et les lymphocytes T cytotoxiques, mais également de l'action de la thrombopoḯtine (TPO), dont la concentration est anormalement faible. La TPO, qui constitue le facteur de croissance principal des mégacaryocytes, est produite de façon basale par le foie, sa fraction libre étant régulée à la fois par la masse mégacaryocytaire et par la masse de plaquettes gagnant la circulation sanguine. Ces deux paramètres sont normaux au cours du PTI, la thrombopénie résultant principalement de la diminution de la durée de vie des plaquettes. Les taux de TPO ne sont donc pas augmentés, et la destruction périphérique des plaquettes n'est pas compensée par une augmentation de la production médullaire.

\section{Causes de l'échec du rituximab}

L'implication de lymphocytes B autoréactifs dans la physiopathologie du PTI, comme dans celle d'autres maladies auto-immunes, a conduit à l'utilisation d'anticorps monoclonaux thérapeutiques comme le rituximab (dirigé contre l'antigène CD20 exprimé par les lymphocytes B) afin d'entraîner leur déplétion. Cependant, $60 \%$ des patients ayant un PTI ne sont pas améliorés par ce traitement. Les données physiopathologiques et marqueurs prédictifs de réponse ne sont pas encore clairement identifiés, seuls une durée d'évolution courte de la maladie et un jeune âge ayant été associés à une meilleure réponse au rituximab $[8,9]$. Plusieurs mécanismes pourraient conduire à son inefficacité. Tout d'abord, alors qu'une déplétion complète en lymphocytes B, à la fois sanguine et splénique, est obtenue après rituximab, la proportion de plasmocytes spléniques (cellules différenciées de la lignée B qui synthétisent les immunoglobulines) est accrue chez les sujets non répondeurs [5]. N'exprimant que faiblement le CD20, les plasmocytes de longue durée de vie représentent la population lymphocytaire B splénique résiduelle chez les patients en échec de rituximab [10]. Sécrétant des anticorps anti-plaquettes, ils participent au maintien de l'activité de la maladie (Figure 2).

D'autre part, le déséquilibre de la balance pro-inflammatoire/antiinflammatoire représentée par le rapport entre lymphocytes $T$ CD $4^{+}$Thl et Treg [5] favorise probablement l'activation des lymphocytes T CD $8^{+}$spléniques. $\varepsilon n$ effet, chez les patients en échec de rituximab, la proportion de lymphocytes $\mathrm{T} C \mathrm{CD} 8^{+}$spléniques exprimant un phénotype effecteur mémoire $C D 27^{-} \mathrm{CD} 28^{-}$ CD62L'CCR7- est accrue. Par ailleurs, ceux-ci expriment fortement la protéine

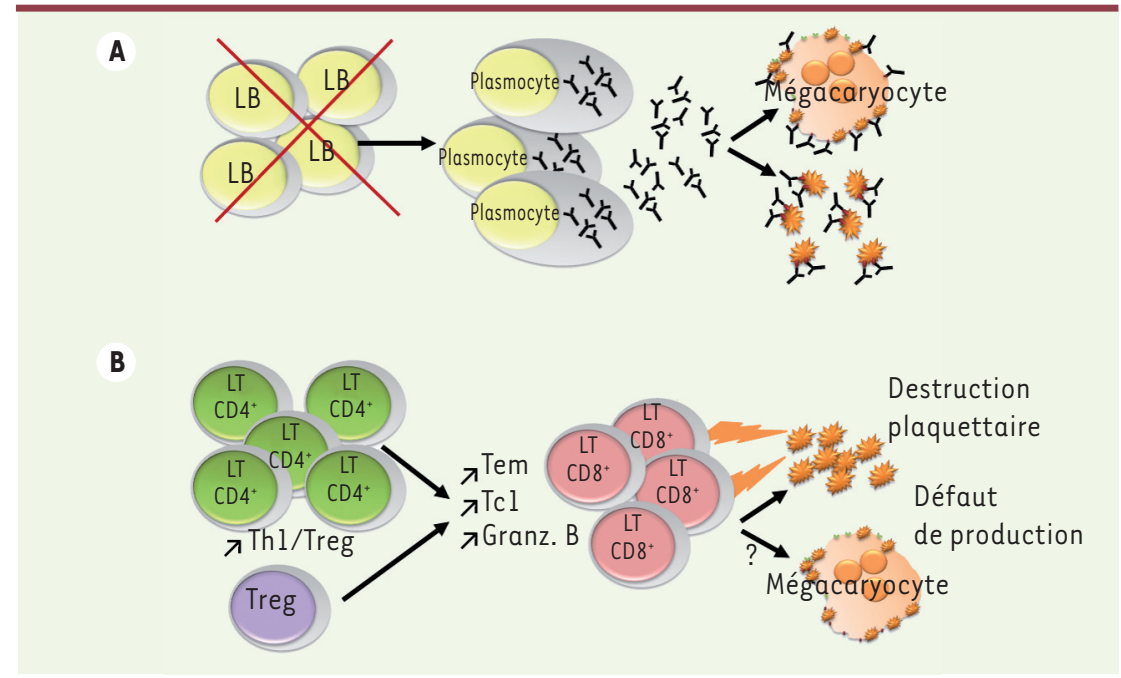

Figure 2. Causes d'échec du traitement par rituximab au cours du PTI. A. Malgré la déplétion lymphocytaire $B$ obtenue après traitement par rituximab, la persistance de plasmocytes à longue durée de vie sécrétant des anticorps anti-GPIIb/IIla, glycoprotéines exprimées par les plaquettes et les mégacaryocytes, participe à la destruction périphérique des plaquettes et à leur défaut de production médullaire. B. Chez certains patients, la thrombopénie résulte de l'implication préférentielle des lymphocytes $T C D 8^{+}$cytotoxiques (Tcl) : ceux-ci ne sont pas ciblés par le rituximab, ce qui explique l'inefficacité de la thérapeutique. Granz. B : granzyme B ; Tem : lymphocytes T CD $8{ }^{+}$effecteurs mémoires. 
cytotoxique granzyme $B$ et la cytokine pro-inflammatoire IFN- $\gamma$ (interféron $\gamma$ ) (Figure 3B) [6]. Enfin, la restriction de la diversité de leur TCR ( $T$ cell receptor) témoigne de la reconnaissance privilégiée de certains antigènes qui, dans ce contexte, sont probablement d'origine plaquettaire, bien que cela reste à démontrer (Figure $3 B$ ). Ainsi, l'ensemble de ces résultats constituent un argument fort pour l'implication des lymphocytes $\mathrm{T} \mathrm{CD}^{+}$spléniques dans la destruction périphérique des plaquettes, en particulier chez les patients qui ne répondent pas au rituximab. Cette cytotoxicité contre les plaquettes, démontrée avec des lymphocytes T CD8 ${ }^{+}$ sanguins [7], pourrait avoir lieu préférentiellement dans la rate, notamment au sein de la pulpe rouge où le contact étroit - nécessaire aux effecteurs lymphocytaires T CD $8^{+}$pour exercer leur cytotoxicité sur leur cible plaquettaire - est possible. Ainsi, l'échec du rituximab pourrait être expliqué par une maladie plutôt dépendante des lymphocytes T $\mathrm{CD}^{+}$(Figure 2). Le PTI, comme probablement d'autres maladies auto-immunes, pourrait donc être une maladie hétérogène, conséquence de deux mécanismes physiopathologiques différents : soit une réponse $B$ anticorps prédominante, soit une réponse $\mathrm{T} C D 8^{+}$prépondérante. Cependant, ces nouvelles connaissances physiopathologiques, acquises grâce à l'étude de la réponse immunitaire splénique, n'ont pas permis pour l'instant de déterminer de nouveaux marqueurs prédictifs de la réponse à ce traitement. $\varepsilon$ n effet, l'analyse de la polarisation des lymphocytes $T$ cytotoxiques circulants et l'expression de granzyme B avant traitement ne permettent pas de distinguer les patients répondeurs des non répondeurs, ce qui témoigne de la localisation préférentiellement splénique de la réponse immunitaire auto-immune au cours du PTI. $\diamond$

Immunologic mechanisms involved in rituximab failure during adult immune thrombocytopenia

A
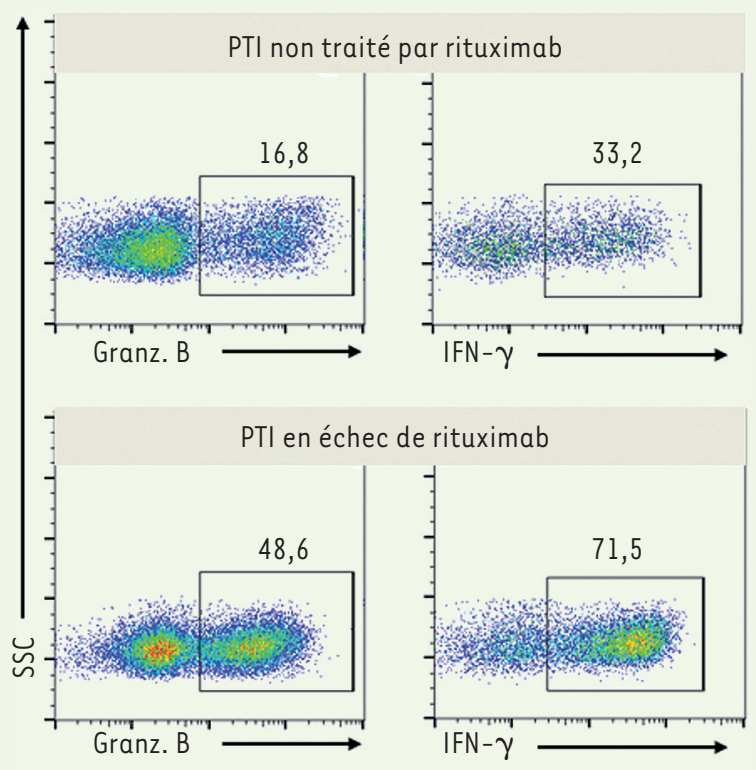

de rituximab

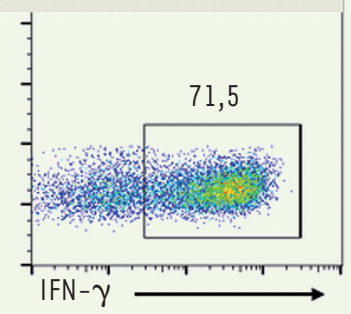

B

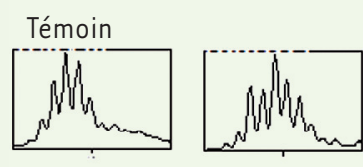

PTI non traité par rituximab
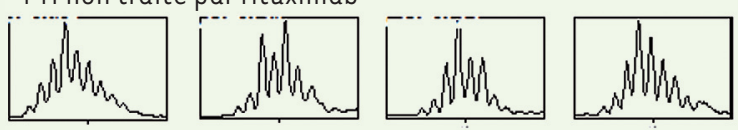

PTI en échec de rituximab

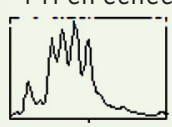

TCRBV4

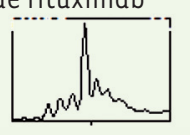

TCRBV8

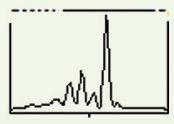

TCRBV14

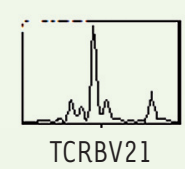

Figure 3. Anomalies des lymphocytes $T C D 8^{+}$spléniques chez les patients en échec de traitement par rituximab. $A$. Les lymphocytes $T C D 8^{+}$spléniques des patients qui ne répondent pas au traitement par rituximab expriment de façon accrue le granzyme B (Granz. B) et l'interféron- $\gamma$ (IFN- $\gamma$ ) comparativement aux sujets non traités. Graphiques de cytométrie en flux, après sélection de la population lymphocytaire $T \operatorname{CD} 8^{+}$(cadre). B. L'analyse du répertoire du récepteur des lymphocytes $T$ (TCR) $C D 8^{+}$spléniques montre une restriction chez les patients en échec de rituximab. La diversité du répertoire du TCR se traduit par une distribution gaussienne des pics qui correspondent à la longueur des différentes régions hypervariables du récepteur (distribution observée chez les témoins). Lorsqu'il existe une restriction du nombre de pics, c'est-à-dire du répertoire, on parle de profil oligoclonal (TCRBV8, 14 et 21 chez les patients en échec de rituximab). L'existence d'un seul pic traduit la présence d'une population monoclonale, ce qui peut s'observer au cours des hémopathies malignes, ce qui n'est pas le cas ici.

\section{LIENS D'INTÉRÊT}

Les auteurs déclarent n'avoir aucun lien d'intérêt concernant les données publiées dans cet article.

\section{RÉFÉRENCES}

1. Cartron G, Rossi JF. Anticorps monoclonaux therapeutiques en oncohematologie. Med Sci (Paris) $2009 ; 25: 1085-9$.
2. Godeau B, Porcher R, Fain 0, et al. Rituximab efficacy and safety in adult splenectomy candidates with chronic immune thrombocytopenic purpura: results of a prospective multicenter phase 2 study. Blood 2008 ; 112 : 999-1004.

3. Semerano L, Boissier MC. Les anticorps monoclonaux dans les maladies immunes inflammatoires chroniques. Med Sci (Paris) 2009 ; 25 : 1108-12.

4. Audia S, Lorcerie B, Godeau B, Bonnotte B. Physiopathologie du purpura thrombopénique immunologique. Rev Med Interne 2011 ; 32 : 350-7. 


\section{RÉFÉRENCES}

5. Audia S, Samson M, Guy J, et al. Immunologic effects of rituximab on the human spleen in immune thrombocytopenia. Blood $2011 ; 118: 4394-400$.

6. Audia S, Samson M, Mahevas M, et al. Preferential splenic $C D 8^{+} T$ cell activation in rituximab-nonresponder patients with immune thrombocytopenia. Blood 2013; 122 : 2477-86.
7. Olsson B, Andersson PO, Jernas M, et al. T-cellmediated cytotoxicity toward platelets in chronic idiopathic thrombocytopenic purpura. Nat Med 2003 ; 9 : 1123-4

8. Arnold DM, Dentali F, Crowther MA, et al. Systematic review: efficacy and safety of rituximab for adults with idiopathic thrombocytopenic purpura. Ann Intern Med $2007 ; 146: 25-33$.

\section{NOUVELLE}

\section{L'aspartate}

Un acide aminé essentiel à la physiologie et à la virulence du bacille de la tuberculose

Alexandre Gouzy ${ }^{1,2}$, Yannick Poquet ${ }^{1,2}$, Olivier Neyrolles ${ }^{1,2}$
> Mieux comprendre les mécanismes d'acquisition et d'assimilation des nutriments par les pathogènes bactériens au sein de leur hôte pourrait permettre d'identifier des voies métaboliques pouvant être inhibées à l'aide de nouveaux antibiotiques.

Mycobacterium tuberculosis, I'agent de la tuberculose, est un pathogène intracellulaire facultatif qui se multiplie à l'intérieur des macrophages dans lesquels il réside, dans un compartiment (le phagosome) où les sources de nutriments sont peu disponibles. Plusieurs études ont permis de mettre en évidence la capacité de $M$. tuberculosis d'exploiter de nombreuses sources de carbone, telles que les triglycérides et le cholestérol, pour proliférer au sein de son hôte [1, 2]. L'azote, comme le carbone, entre dans la composition de biomolécules majeures telles que les acides aminés ou les nucléotides, et constitue un élément essentiel à la vie. Cependant, contrairement aux besoins en carbone, peu de choses sont connues en ce qui concerne les besoins en azote des pathogènes intracellulaires, et de M. tuberculosis en particulier, au cours de l'infection. Nous avons pu récemment mettre en évidence qu'un acide aminé, l'aspartate, constituait une source majeure d'azote utilisée par la bactérie pour infecter son hôte [3].

\section{L'aspartate est accessible}

à M. tuberculosis au sein des macrophages

Il est intéressant de se demander comment les nutriments, comme l'aspartate, ont accès au phagosome de M. tuberculosis. En utilisant une technique dite de NanoSIMS (secondary ion mass spectrometry), associant l'imagerie à la spectrométrie de masse, nous avons pu montrer que cet acide aminé s'accumule au sein des phagosomes renfermant le bacille (Figure 1). L'aspartate entre dans des cellules de mammifères grâce à des transporteurs de la superfamille des solute carriers (SLC), en particulier ceux appartenant à la famille des transporteurs de haute affinité du glutamate et des acides aminés neutres (SLCl) et des transporteurs d'acides aminés cationiques (SLC7). Parmi ces transporteurs, nous avions montré que le transporteur d'aspartate SLC1A2 est exprimé fortement par des macrophages humains en réponse à une infection par M. tuberculosis [4]. L'augmentation de l'expression de ce transporteur pourrait ainsi
9. Mahevas M, Ebbo M, Audia S, et al. Efficacy and safety of rituximab given at $1000 \mathrm{mg}$ on days 1 and 15 compared to the standard regimen to treat adult immune thrombocytopenia. Am J Hematol 2013 ; 88 : 858-61.

10. Mahevas M, Patin P, Huetz F, et al. B cell depletion in immune thrombocytopenia reveals splenic long-lived plasma cells. J Clin Invest $2013 ; 123$ : 432-42.
${ }^{1}$ Centre national de la recherche scientifique (CNRS), UMR 5089, Institut de pharmacologie et de biologie structurale (IPBS), IPBS, 205, route de Narbonne, 31077 Toulouse Cedex 04, France ;

2 Université de Toulouse, Université Paul Sabatier, IPBS, Toulouse, 31077, France. olivier.neyrolles@ipbs.fr

favoriser la multiplication du bacille tuberculeux à l'intérieur des cellules, ce qui reste à déterminer.

\section{Mycobacterium tuberculosis utilise le transporteur AnsP1 pour capturer} l'aspartate

Grâce à une approche de mutagenèse dirigée, nous avons pu montrer que l'import de l'aspartate chez M. tuberculosis est assuré par un transporteur unique, appelé AnsPl, et que ce transporteur est requis pour la multiplication du bacille chez l'animal. II est surprenant de constater que AnsPl appartient à la classe des transporteurs d'acides aminés originalement prédits pour transporter l'asparagine, et ne possède pas d'homologie avec les transporteurs d'acides aminés dicarboxyliques classiquement impliqués dans le transport de l'aspartate. Un homologue de AnsP1, appelé AnsP2, avec lequel AnsP1 partage un niveau élevé de similitude ( $83 \%$ ), existe chez M. tuberculosis, mais ne compense pas la fonction de AnsPl pour l'import d'aspartate. La spécificité unique d'AnsPl pour l'aspartate suggère une évolution sélective de ce transporteur qui a pu conférer un avantage au pathogène pour sa multiplication 